\title{
Changes in maternal volumetric bone mineral density in the peripheral skeleton during pregnancy as determined by single-slice peripheral Quantitative Computed Tomography
}

\author{
M.Ó. Breasail ${ }^{1}$, A. Prentice ${ }^{1}$ and K. Ward ${ }^{1,2}$ \\ ${ }^{1}$ Medical Research Council Elsie Widdowson Laboratory (MRC EWL), CB1 9NL and ${ }^{2}$ Medical Research Council \\ Lifecourse Epidemiology Unit (MRC LEU), University of Southampton, SO16 6 YD.
}

At birth the fetus contains 25-30 g of calcium (Ca), although whether the maternal skeletal stores of calcium are a source (in addition to increased intestinal absorption and reduced excretion) is not fully known. There is limited evidence from DXA studies before and shortly after pregnancy suggesting some mobilisation of maternal Ca stores ${ }^{(1,2)}$; such mobilisation has been consistently observed during lactation ${ }^{(1,3-5)}$. Peripheral QCT scanners measure volumetric bone mineral density (vBMD) and geometry in the appendicular skeleton. To determine if pregnancy induced bone mineral changes occur in the appendicular skeleton we scanned pregnant women at the distal tibia and radius at two time points during pregnancy (14-16 weeks and 34-36 weeks). Healthy premenopausal non-pregnant non-lactating controls were also scanned with a similar length between visits.

Data were obtained from 90 (53 pregnant) healthy premenopausal women (mean age 35.4 (SD 3.8) years). Scans with pQCT (XCT $2000 \mathrm{~L}$, Stratec) were obtained at $4 \%$ of the length of the lower leg and forearm. Independent t-tests tested for unadjusted differences between change in vBMD. Linear regression models were then fitted for total and trabecular vBMD and total cross-sectional area (CSA) at the radius and tibia with the terms: (1) height, baseline weight, group and (2) model 1 plus age, weight change, parity, ever smoked and previous contraception use. In each case the model of best fit was selected using backwards stepwise regression for each outcome measure of interest. Data are presented from model 2.

At baseline there were no statistically significant difference between the two groups $(p<0 \cdot 05)$. Between visits the pregnant participants gained 9 (SD 3.4) kg. There were significant decreases in the pregnant group compared to non-pregnant controls of: total vBMD $1.8 \%($ SE 0.9) $(\mathrm{P}<0.001)$ and in trabecular vBMD $1.4 \%(\mathrm{SE} 0.5)(\mathrm{P}<0.001)$. These decreases exceed the precision error of the scanning technique. No significant changes in tibia total CSA. Testing at the distal radius found no statistically significant changes during pregnancy in any of the measured parameters of interest.

During pregnancy bone mineral is mobilised from the distal tibia, a trabecular rich site. In contrast there appeared to be conservation of mineral at the distal radius during pregnancy. The basis for the site differences requires further investigation but is consistent with previous DXA studies.

1. Olausson H, Laskey MA, Goldberg GR et al. (2008) Am J Clin Nutr 88, 1032-1039.

2. Kaur M, Pearson D, Godber I et al. (2003) Bone 32, 449-454.

3. Laskey MA \& Prentice A (1997) Lancet 349, 1518-1519.

4. Laskey MA, Prentice A, Hanratty LA et al. (1998) Am J Clin Nutr 67, 685-692.

5. Laskey MA \& Prentice A (1999) 94, 608-615. 